\title{
Title Correction: Transcarotid Mechanical Thrombectomy for Embolic Intracranial Large Vessel Occlusion after Endovascular Deconstructive Embolization for Carotid Blowout Syndrome
}

Chi-Ju Lu, MD, Yen-Heng Lin, MD, MS, Chung-Wei Lee, MD, PhD

Department of Medical Imaging, National Taiwan University Hospital, Taipei, Taiwan

Correction to: Neurointervention https://doi.org/10.5469/neuroint.2019.00241, published on March, 2020, Neurointervention 2020;15:37-43.

The originally published version of this article contained an error in the title. In the title of this article, the word "Deconstructice" should be corrected "Deconstructive".

The author apologizes for any inconvenience that it may have caused.

Before correction: Transcarotid Mechanical Thrombectomy for Embolic Intracranial Large Vessel Occlusion after Endovascular Deconstructice Embolization for Carotid Blowout Syndrome

After correction: Transcarotid Mechanical Thrombectomy for Embolic Intracranial Large Vessel Occlusion after Endovascular Deconstructive Embolization for Carotid Blowout Syndrome 